\title{
Optimal Distance Between Wells In SAGD Based On Material Balance Method
}

\author{
A.Ya. Gilmanov \\ Institute of Physics and Technology \\ University of Tyumen \\ Tyumen, Russia \\ agilmanov1996@gmail.com
}

\author{
A.P. Shevelev \\ Institute of Physics and Technology \\ University of Tyumen \\ Tyumen, Russia \\ alexandershevelev@mail.ru
}

\begin{abstract}
The article discusses the modeling of SAGD using the method of material balance and Butler's model. This method is very important in the development of heavy oil fields. The application of the integral approach based on the material balance method and Butler's model allows saving time of modeling without loss of accuracy, giving physically consistent results. For the first time in such integral approach, debits of water and oil are considered to be variable. Real data on Western fields are used for calculations. The final results are comparable with similar data on foreign articles, which are, however, calculated using the basic system of equations of mechanics of multiphase systems, requiring large time-consuming. The key result is an optimal distance between the wells, allowing the development of real fields with maximum economic benefit.
\end{abstract}

Keywords-physical and mathematical modeling, oil recovery, steam-assisted gravity drainage, underground hydromechanics, numerical methods, heat and mass transfer.

\section{INTRODUCTION}

Currently, the share of hard-to-recover oil reserves is quite large among all oil reserves and increases over time. In the development of such fields, common thermal methods are not always economically effective to use. World experience shows that heavy oil fields are successfully developed using the technology of Steam Assisted Gravity Drainage (SAGD), there is also experience in the development of similar fields in Russia. In addition, modeling of SAGD is important for maximum heating of the reservoir and oil recovery. The existing and well-proven approach based on the basic system of equations of mechanics of multiphase systems requires a lot of time and a lot of field data, which is expensive or difficult and not always possible to have.

Therefore, the aim of this work is to apply the integral approach of modeling of SAGD based on the method of material balance and Butler's model, which allows calculating the basic parameters of SAGD quickly and without loss of accuracy, without requiring a large amount of data. Unlike the previous similar model, the flow rates of water and oil are considered to be variable, which is consistent with the real data. The most important practically significant result is finding the optimal distance between wells for the most efficient field development.
In addition, the water content of oil products is calculated to estimate the time of operation of the well.

\section{TECHNOLOGY OF SAGD}

In the 80-ies of the $\mathrm{XX}$ century, with significant investment in research projects in the field of thermal methods, as well as with the development of the technology of horizontal drilling in Canada the technology of steam-assisted gravity effects with the use of a pair of horizontal wells was developed, better known in the global industry as SAGD (Steam Assisted Gravity Drainage).

This technology requires the drilling of two horizontal wells in length, as a rule, from $800 \mathrm{~m}$ to $1000 \mathrm{~m}$, located one above the other, usually at a distance of $5 \mathrm{~m}$. In the classic version of the upper well is steam injector, the lower well is producer.

The process begins with the preparatory stage of preheating, during which steam circulates in both wells, respectively; oil production is not conducted. Due to the heat transfer, the formation zone between wells is heated, which leads to a decrease in the viscosity of heavy oil.

At the main stage [1], the steam enters through the injection well located above the extraction well; it breaks into the upper part of the productive formation, since its density is lower than that of the reservoir contents. The steam chamber, increasing in size, is formed, on the surface of which the heat exchange process takes place with the oil-saturated zone. As a result, the vapor condenses and cools gradually, together with the heated oil flowing down to a producing well under the influence of gravity. The steam chamber grows up to reach the top of formation, then this chamber grows to the sides, constantly in contact with the oil, whereby the heat loss is low enough with this method. Other advantages of the method are simplicity of realization, high flow rate and coefficient of extraction of viscous oil as a result of its application, at the same time, unlike the method of steam-cyclic influence on bottom-hole formation zone, oil is extracted continuously. Disadvantages of SAGD technology are the connection of a large part of the cost of oil production with the cost of steam generation, the requirement of a large water source, as well as the need for water treatment equipment with high thickness, in 
addition, for the effective application of the technology it requires a homogeneous layer of relatively high thickness.

In [2], the following subspecies of SAGD are described: ES-SAGD, SAS, SAP and SAGD with the use of the mechanism of evaporation-condensation (VCM, vaporizingcondensing mechanism). Their characteristic is given.

In the case of ES-SAGD (expanding solvent SAGD, a mechanism of vapor-gravity action with a spreading solvent), a solvent in the vapor phase of low concentration is introduced together with steam in a process where gravity plays a major role. This solvent condenses on the border of the steam chamber, reducing the viscosity of the oil not only because of its heating, but also because of its dissolution. Instead of a solvent can be used foam, then the mechanism is called FASAGD (foam assisted SAGD, SAGD with the use of foam). As a result, the viscosity of the oil decreases more strongly than with conventional SAGD, but additional cleaning of the oil from the solvent is necessary.

General scheme of SAS (steam-alternative-solvent, an alternative mechanism with solvent and steam) is similar to the ES-SAGD, but the steam and solvent are injected not constantly, but for a certain period of time with rapid pump via the injection well.

SAP (solvent aided process, a process using solvents) is a kind of mechanism of steam assisted gravity drainage spreading solvent, where for the solvent a small amount of light hydrocarbons is used.

The viscosity of the oil decreases slightly and its mobility increases using the vaporizing-condensing mechanism of light oil fractions together with SAGD on the edges of the oil-vapor interface. As the temperature difference increases, the vaporized light fractions condense on the surface of the oilvapor partition, which reduces the viscosity and density of the oil at the front of its movement. When the pressure of steam injection increases, its temperature increases, which increases the efficiency of the method. High viscous oil production also increases.

\section{PHYSICAL AND MATHEMATICAL CALCULATIONS}

There is a general approach based on the fundamental system of equations of mechanics of multiphase systems, including the equations of conservation (transformation) of mass, momentum, energy for a multi-component system, as well as various closing ratios [1], [3], [4], [5].

The advantages of this method are a very detailed description of the process of steam-gravity drainage, taking into account any effects, and the fact that this system of equations has been tested for years, but, unfortunately, modeling by this method requires a lot of time. In addition, this approach requires a significant amount of data, including detailed descriptions of the various interfacial interactions, which are usually not available, and obtaining such information requires large time, technological and financial costs.

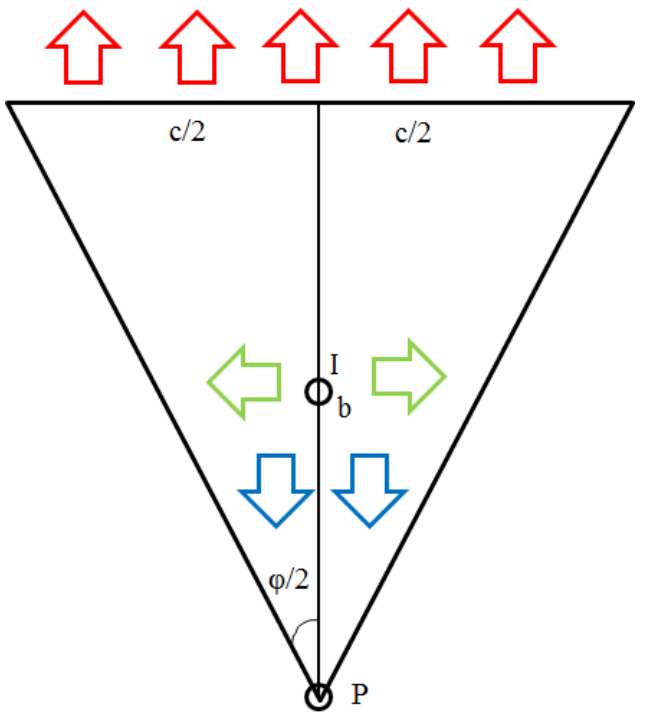

Fig. 1. Distribution of the heated area of the Butler's model in the cross section. I is injection well, $\mathrm{P}$ is producing well. Red arrows mean heat transfer by Newton-Richmann law, green arrows mean steam injection, blue arrows mean heat flow of water and oil.

To overcome these difficulties, you can use the method of material balance. This is an approach based on the basic conservation laws without taking into account all the effects in detail. The main advantage of this method is that it is simple and easy to use. However, it is important to avoid significant loss of accuracy by obtaining physically consistent results. Therefore, for a correct description of the SAGD process using the method of material balance, the model proposed by Butler should be used [1], [6]. This model is simplified and based on generalization of experimental data. However, the Butler model was first proposed for vertical wells, and its verification is necessary, which will be done in this work.

According to this model, when the steam is pumped through the injection well, it gradually rises in the reservoir due to the fact that the heated oil flows down to the production well under the influence of gravitational forces. In addition, the steam chamber extends to the sides. For a long horizontal well, a wedge in the volume and an isosceles triangle in the section are formed as a result. The injection well is located somewhere at the height $b$ of the triangle (often in the middle, but not necessarily), producer is located in its top (Fig. 1), where $b$ is the reservoir thickness, $c$ is the distance between the well pairs; $\varphi$ is the total angle of the cross section. Heat transfer to the top of the reservoir, located above the base of the wedge, goes according to Newton-Richman's law. This model is quite simple in mathematical description and is a good approximation of the real heating of the reservoir under steam-gravity drainage [1].

There is also a cone approximation instead of a wedge, but it shows good data only for a well as a point source rather than a long horizontal pipe.

Heat balance, which is obtained from the sum of the equations for the balances of the corresponding phases: 
$d\left(m V(t)\left(S_{w}(t) C_{w}\left(T_{s}-T_{o}\right) \rho_{w}+S_{s}(t) l \rho_{s}+\left(1-S_{w}(t)-S_{s}(t)\right) C_{o}\left(T_{s}-\right.\right.\right.$ $\left.\left.\left.T_{o}\right) \rho_{o}\right)\right) / d t=Q_{s} l-2 d_{w} \alpha A(t)\left(T_{s}-T_{o}\right) / b-Q_{o}(t) C_{o}\left(T_{s}-T_{o}\right)-Q_{w}(t) C_{w}\left(T_{s}-\right.$ $\left.T_{o}\right)$,

where the index $w$ denotes water, $s$ is steam, $o$ is oil; $m$ is the porosity, $V$ is the volume of the heated area, $t$ is time, $S_{j}$ is the $j$-th phase volume saturation, $C_{j}$ is the specific heat capacity of the $j$-th phase and $T_{j}$ is the temperature of the $j$-th phase, $\rho_{j}$ is the density of $j$-th phase, $l$ is the latent heat of vaporization of water, $Q_{j}$ is the mass flow rate of the $j$-th phase, $d_{w}$ is the length of the wedge of the heated area, $\alpha$ is the transfer coefficient, $A$ is the cross-sectional area of the heated volume of the model, through which the heat transfer area of the law of Newton-Richman is expressed using simple geometrical ideas.

Mass balance, which is obtained from the sum of the equations for the balances of the corresponding phases, is: $Q_{w}(t)$.

$d\left(m V(t)\left(S_{w}(t) \rho_{w}+S_{s}(t) \rho_{s}+\left(1-S_{w}(t)-S_{s}(t)\right) \rho_{o}\right)\right) / d t=Q_{s}-Q_{o}(t)-$

The following relations are used to close the system of equations (1) - (2). The transition to the consideration of twophase flow:

$$
Q_{w}(t) F(t)+Q_{o}(t)(1-F(t))=Q(t)
$$

where $F$ is a function of saturation, $Q$ is the final rate of oilwater flow.

And the ratio between water saturation and saturation function, which is derived from the view of the BuckleyLeverett function:

$$
S_{w}(t)=S_{w}(F(t))=\mu /(\mu-1+1 / F(t)),
$$

where $\mu$ is the relative viscosity of water to viscosity of the oil after heating.

In addition, the following balance relations are valid for oil and water rates:

$$
Q_{o}(t)=\rho_{o} d\left(\left(1-S_{w}(t)-S_{s}(t)\right) V(t)\right) / d t,
$$

$$
Q_{w}(t)=Q_{s}-\rho_{w} d\left(S_{w}(t) V(t)\right) / d t .
$$

The model layer with the following characteristics, close in size to the data on Western fields, where SAGD technology has found the greatest application and has a long experience of its use, was taken for calculations [2], [7], [8], [9]:

$$
\begin{gathered}
Q_{s}=2.34 \mathrm{~kg} / \mathrm{s}, \alpha=1.5 \mathrm{~W} /\left(\mathrm{m}^{2} \cdot \mathrm{K}\right), C_{w}=4200 \mathrm{~J} /(\mathrm{kg} \cdot \mathrm{K}), \\
C_{o}=4500 \mathrm{~J} /(\mathrm{kg} \cdot \mathrm{K}), b=30 \mathrm{~m}, d_{w}=100 \mathrm{~m}, \mathrm{~m}=0.33, \\
\rho_{o}=850 \mathrm{~kg} / \mathrm{m}^{3}, \rho_{w}=1000 \mathrm{~kg} / \mathrm{m}^{3}, \rho_{s}=100 \mathrm{~kg} / \mathrm{m}^{3}, \\
l=2 \cdot 10^{6} \mathrm{~J} / \mathrm{kg}, T_{s}=478 \mathrm{~K}, T_{o}=288 \mathrm{~K}, \mu=0.1 .
\end{gathered}
$$

The system of equations (1) - (6) is solved numerically using finite difference scheme. The number of time steps is assumed to be 2000. In addition, the known geometric relationships are used.

The relationship of the volume of the wedge on the $i$-step with cross-sectional area and the length of the wedge:

$$
V_{i}=A_{i} d_{w}
$$

the opening angle of the cross section:

$$
\varphi_{i}=2 \operatorname{arctg}\left(A_{i} / b^{2}\right)
$$

In addition, the rate of the two-phase flow:

$$
Q(t)=Q_{o}(t)+Q_{w}(t)
$$

The share of water in production:

$$
W(t)=Q_{w}(t) / Q(t)
$$

The following initial conditions [7], [10] are selected: initial time step $t=0$, time step $\Delta t=72000 \mathrm{~s}, A_{l}=V_{l}=0, S_{w l}=0.2$, $S_{s l}=0, Q_{o l}=0.78 \mathrm{~kg} / \mathrm{s}, Q_{w l}=0.195 \mathrm{~kg} / \mathrm{s}$.

First (5) and (6) are substituted in (1) - (3), taking into account the approximation of the finite-difference scheme and (9), then from (3) is expressed $F$ and substituted in (4), from the resulting equation is expressed volume on the $i+1$ step $V_{i+1}$, which is substituted in (1) and (2). After that (2) is solved by simple integration, $S_{S(i+1)}$ is expressed from the obtained result, which is substituted in (1), taking into account (7).

The final equation is:

$$
A_{y i} S_{w(i+1)}^{2}+B_{y i} S_{w(i+1)}+C_{y i}=0,
$$

where coefficients:

$$
\begin{gathered}
A_{y i}=A_{x 2 i}\left(\left(m\left(\rho_{s}-\rho_{o}\right)-\rho_{o}\right)\left(C_{o}\left(T_{s}-T_{o}\right) \rho_{o}-C_{w}\left(T_{s}-T_{o}\right) \rho_{w}\right)+\left(C _ { o } \left(T_{s}-\right.\right.\right. \\
\left.\left.\left.T_{o}\right) \rho_{o}-l \rho_{s}\right)\left((1-m) \rho_{w}+(1+m) \rho_{o}\right)\right)+\left(Y_{i}+Q_{s} l \Delta t / m-Z_{i}\right)(((1- \\
\left.\left.m) \rho_{w}+(1+m) \rho_{o}\right)+(\mu+1)\left(m\left(\rho_{s}-\rho_{o}\right)-\rho_{o}\right)\right),
\end{gathered}
$$

$B_{y i}=A_{x 2 i}\left(\rho_{o}(1+m) l \rho_{s}\right)+\mu A_{x l i}\left(C_{w}\left(T_{s}-T_{o}\right) \rho_{w}\left(m\left(\rho_{s}-\rho_{o}\right)-\rho_{o}\right)+(1-\right.$ m) $\left.\rho_{w} l \rho_{s}+(1+m) \rho_{o} l \rho_{s}-C_{o}\left(T_{s}-T_{o}\right) \rho_{o}\left(m\left(\rho_{s}-\rho_{o}\right)+(1-m) \rho_{w}+m \rho_{o}\right)\right)-$ $\left(Y_{i}+Q_{s} l \Delta t / m-Z_{i}\right)\left((1+m) \rho_{o}+(\mu+1)\left(m\left(\rho_{s}-\rho_{o}\right)-\rho_{o}\right)\right)$,

$$
C_{y i}=\mu A_{x l i}\left(m \rho_{s} C_{o}\left(T_{s}-T_{o}\right) \rho_{o}-(1+m) \rho_{o} l \rho_{s}\right),
$$

$$
A_{x l i}=Q_{s} \Delta t / \rho_{w}+V_{i} S_{w i}
$$

$$
A_{x 2 i}=\mu Q_{s} \Delta t / \rho_{w}+\mu V_{i} S_{w i}-V_{i}\left(1-S_{w i}-S_{s i}\right),
$$




$$
Y_{i}=V_{i}\left(S_{w i} C_{w}\left(T_{s}-T_{o}\right) \rho_{w}+S_{s i} l \rho_{s}+\left(1-S_{w i}-S_{s i}\right) C_{o}\left(T_{s}-T_{o}\right) \rho_{o}\right),(17)
$$

$$
Z_{i}=\Delta t\left(2 d_{w} \alpha A_{i}\left(T_{s}-T_{o}\right) / b+Q_{o i} C_{o}\left(T_{s}-T_{o}\right)+Q_{w i} C_{w}\left(T_{s}-T_{o}\right)\right) / m . \text { (18) }
$$

From (11), taking into account physical sense and nonnegativity of water saturation, the final expression for it is obtained:

$$
S_{w(i+1)}=\left(-B_{y i}+\left(B^{2}{ }_{y i}-4 A_{y i} C_{y i}\right)^{1 / 2}\right) /\left(2 A_{y i}\right) .
$$

The angle and water content of products are calculated at the end from (8) and (10).

Calculations were carried out using the program in Delphi.

\section{RESULTS AND DISCUSSION}

The final graphs of the dependence of the volume, the total angle of the cross section of the heated region and the water content of the product from the time of heating are shown in Fig. 2, Fig. 3, Fig. 4. Also, Fig. 5 shows a graph of the dependence of the area of the cross section of the wedge on the time of heating. It can be seen that the obtained graphs are stable.

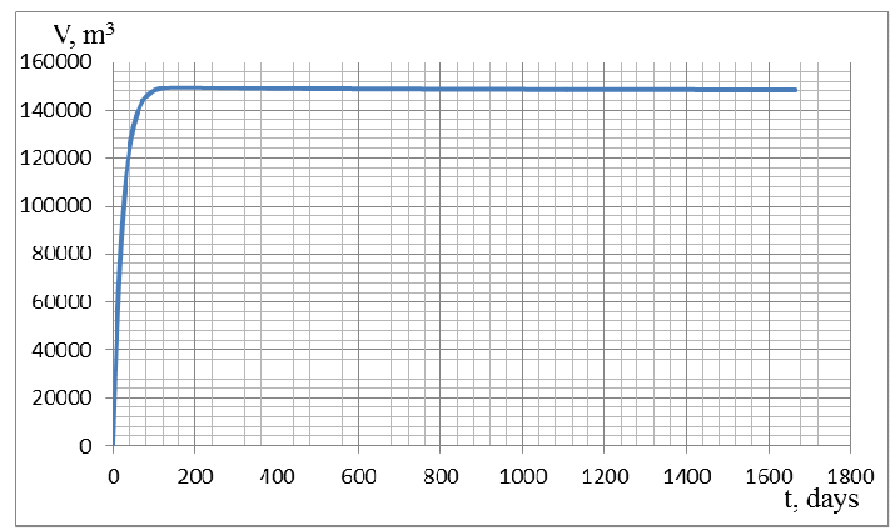

Fig. 2. Volume of heated area depending on the time of heating.

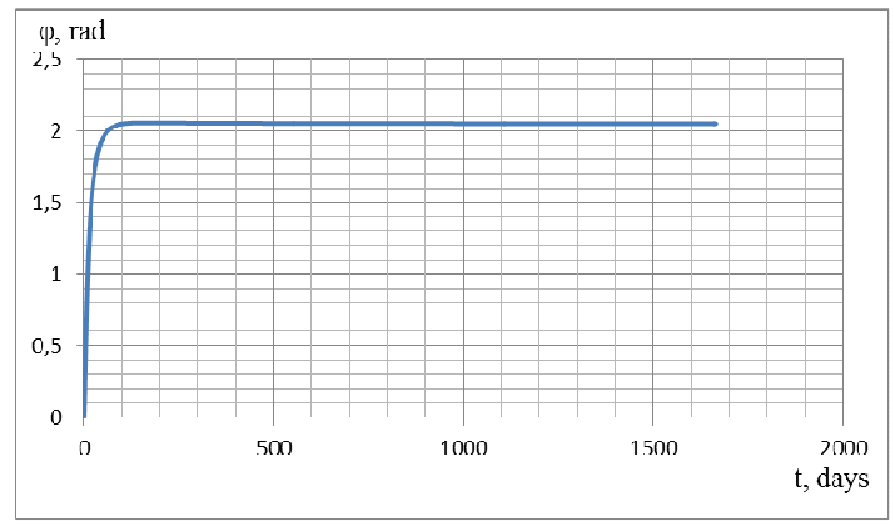

Fig. 3. Full opening angle of the cross section of heated area depending on the time of heating.

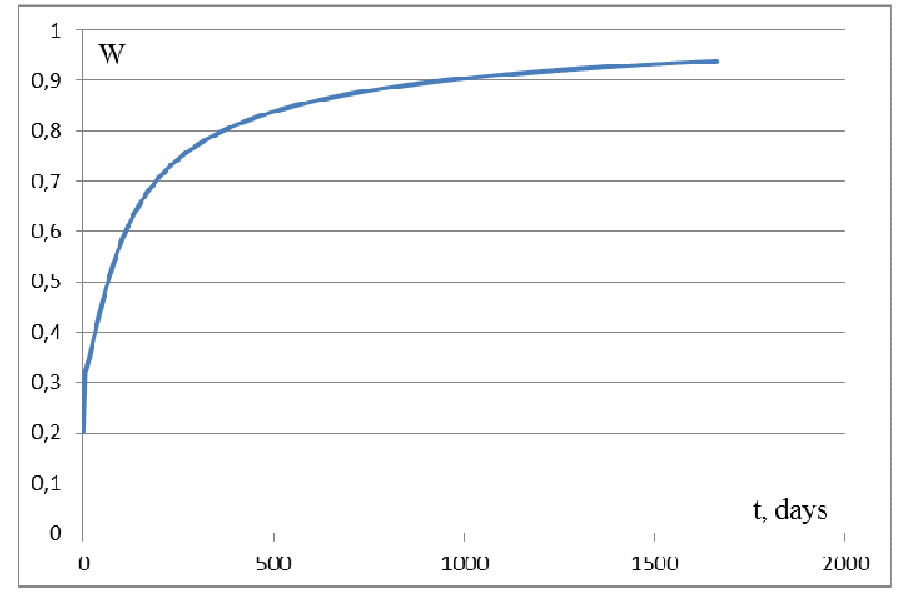

Fig. 4. Dependence of water content of production on the time of heating.

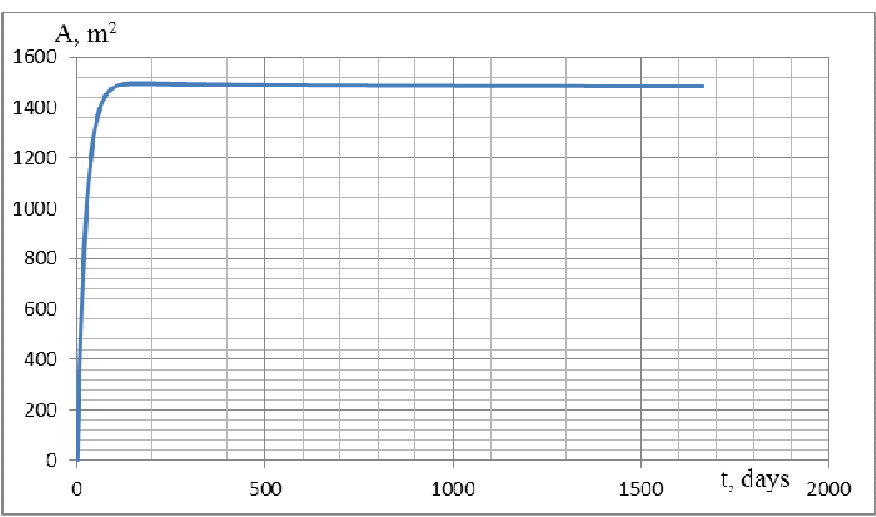

Fig. 5. Area of the cross section of wedge depending on the time of heating.

It is important to add that the distance between wells $c$ is easy to determine from the geometry using the maximum angle $\varphi_{\max }$ from the graphs:

$$
c / 2=\operatorname{btg}\left(\varphi_{\max } / 2\right)
$$

$$
c=2 b \operatorname{tg}\left(\varphi_{\max } / 2\right) \text {. }
$$

The maximum angle is taken equal to 2.05.

The distance between the wells is calculated in such a way that the steam chambers from different well pairs do not overlap each other.

Thus, the obtained graphs prove the physical consistency of the model, because of increasing of the volume, area and angle of the cross section of the heated area over time, after which, when a certain point in time (the time of heating), the growth of these characteristics ceases. This happens when the equalization of the heat loss with the injected with water heat. The time of heating is about 80 days. After heating, in order to maintain the maximum volume of the SAGD area, it is necessary to continue steam injection and operation of this pair of wells until the water content of the products reaches the 
values of 0.95 or more (the values are determined by the one who operates the field on profitability), which occurs after 1500 days (about 4 years) and is a real value for typical fields. The increase of water content of production also slows down over time. Also from (21) the distance between wells is $100 \mathrm{~m}$, which coincides well with the real data for SAGD for Western fields [2]. This proves the applicability of the proposed model for all future versions of SAGD.

\section{CONCLUSIONS}

1. It is determined that the proposed integral model based on the method of material balance and the model of the Butler, in which for the first time the production rates of oil and water are considered to be variables, based on real field data allows you to quickly calculate the SAGD process with good accuracy.

2. The obtained results are quantitative and qualitatively similar to the real data on Western fields, the distance between the wells is about $100 \mathrm{~m}$ with the corresponding initial data.

3. The physical consistency of the model is proved by stopping the growth of the volume and cross-sectional angle of the warmed-up area, when the heat pumped with steam is equal to the heat loss. At the same time, the growth of the heated volume itself leads to an increase in the area of high-viscosity oil subjected to heat processing, and, therefore, an increase in the oil recovery factor.

4. The time of heating is about 80 days. After heating, in order to maintain the maximum volume of the SAGD area, it is necessary to continue steam injection and operation of this pair of wells.

5. Field developer should stop the operation of a pair of wells after 1500 days because of the high water content of production, which is also calculated for the first time in this work.
6. The proposed model can be further used to calculate the optimal SAGD parameters for real fields.

7. On the basis of the real data it is shown that the Butler model can be used for horizontal wells, it was possible to confirm the correctness of the model and the integral approach using the method of material balance.

\section{References}

[1] A. Ya. Gilmanov, A. P. Shevelev, Physical and Mathematical Modeling of Steam-Assisted-Gravity-Drainage of Heavy Oil Fields Based on Material Balance Method. Tyumen State University Herald. Physical and Mathematical Modeling. Oil, Gas, Energy, 2017, vol. 3, no 3, pp.5269.

[2] M. Ghasemi, K. H. Whitson, Compositional variation in SAGD. Paper №SPE-175022-MS. Society of Petroleum Engineers, 2015.

[3] W. Xiong, M. Bahonar, Z. Chen, Development of a thermal wellbore simulator with focus on improving heat loss calculations for SAGD steam injection. Article №SPE-174408-MS. Society of Petroleum Engineers, 2015.

[4] T. T. Garipov, D. V. Voskov, H. A. Tchelepi, Rigorous coupling of geomechanics and thermal-compositional flow for SAGD and ESSAGD operations. Paper №SPE-174508-MS. - Society of Petroleum Engineers, 2015.

[5] C. M. Nascimento, Design, optimization and operation of SAGD wells using dynamic flow simulations. Article №SPE-180459-MS. Society of Petroleum Engineers, 2016.

[6] M. Keshavarz, T. G. Harding, Z. Chen, Modification of Butler's unsteady-state SAGD theory to include the vertical growth of steam chamber. Article №SPE-180733-MS. Society of Petroleum Engineers, 2016.

[7] S. M. Farouq Ali, Life after SAGD - 20 years later. Paper №SPE180394-MS. Society of Petroleum Engineers, 2016.

[8] Q. Li, Z. Chen, A new analysis on the convective heat transfer at the edge of the SAGD chamber. Paper №SPE-175063-MS. Society of Petroleum Engineers, 2015.

[9] I. K. Kikoin (ed.), Tables of physical quantities. Handbook. Moscow: Atomizdat, 1976. 1008 pp.

[10] D. Ji, S. Yang, H. Zhong, M. Dong, Z. Chen, L. Zhong, Re-examination of fingering in SAGD and ES-SAGD. Paper №SPE-180708-MS. Society of Petroleum Engineers, 2016. 